\title{
Quasistatic magnetoelectric multipoles as order parameter for pseudogap phase in cuprate superconductors
}

\author{
M. Fechner, ${ }^{1, *}$ M. J. A. Fierz, ${ }^{1}$ F. Thöle, ${ }^{1}$ U. Staub,${ }^{2}$ and N. A. Spaldin ${ }^{1}$ \\ ${ }^{1}$ Materials Theory, ETH Zurich, Wolfgang-Pauli-Strasse 27, 8093 Zürich, Switzerland \\ ${ }^{2}$ Swiss Light Source, Paul Scherrer Institut, CH-5232 Villigen PSI, Switzerland
}

(Received 19 December 2015; published 20 May 2016)

\begin{abstract}
We introduce a mechanism in which coupling between fluctuating spin magnetic dipole moments and polar optical phonons leads to a nonzero ferroic ordering of quasistatic magnetoelectric multipoles. Using firstprinciples calculations within the LSDA $+U$ method of density functional theory, we calculate the magnitude of the effect for the prototypical cuprate superconductor $\mathrm{HgBa}_{2} \mathrm{CuO}_{4}$. We show that our proposed mechanism is consistent, to our knowledge, with all experimental data for the onset of the pseudogap phase and therefore propose the quasistatic magnetoelectric multipole as a possible pseudogap order parameter. Finally, we show that our mechanism embraces some key aspects of previous theoretical models, in particular the description of the pseudogap phase in terms of orbital currents.
\end{abstract}

DOI: 10.1103/PhysRevB.93.174419

\section{INTRODUCTION}

The partial persistence of the superconducting gap above the superconducting transition temperature in the underdoped high- $T_{c}$ cuprate superconductors has long been acknowledged to be key in understanding the nature of pairing in the superconducting state [1]. Following the first observation of its signature in the spin-lattice relaxation rate in nuclear magnetic resonance (NMR) experiments [2], it has been unclear for many years whether this so-called pseudogap region forms a distinct phase, with a line of phase transitions into the neighboring strange-metal phase, or whether doping instead causes a continuous increase in the Fermi surface and a corresponding gradual crossover to strange metallicity. Indeed, the usual signatures of a phase transition, such as changes in known symmetries or singularities in susceptibilities, remain elusive. The importance of the distinction, the phase transition scenario naturally supports the proposed existence of a zerokelvin quantum critical point within the superconducting dome, has motivated intensive and ongoing research.

A variety of often technically challenging experiments, including spin-polarized elastic neutron diffraction [3], ultrasound measurements [4], nuclear magnetic and quadrupolar $(\mathrm{NQR})$ resonance $[5,6]$, and muon spin resonance $(\mu \mathrm{SR})$ [7] now point to the existence of a phase transition, and the focus of attention has shifted to determining the nature of the "hidden" order parameter. The experimental situation regarding the identity of the order parameter is unconverged, with different experiments giving apparently conflicting results, at least when they are interpreted in terms of an ordering that involves magnetic dipole moments. The key findings are as follows.

A clear indication that the pseudogap ordering has a magnetic origin comes from spin-polarized elastic neutron diffraction which finds differences between the non-spin-flip and spin-flip channels below $T^{*}$ in underdoped $\mathrm{YBa}_{2} \mathrm{Cu}_{3} \mathrm{O}_{6+x}$ (YBCO) [3]. For overdoped samples outside of the pseudogap regime, in contrast, the spin-flip and non-spin-flip signals are both flat, indicating zero magnetic intensity at all temperatures.

*michael.fechner@mat.ethz.ch
The observed increase in the spin-flip signal when the neutron polarization is parallel to the wave vector of the $Q=(0,1,1)$ Bragg peak indicates that the time-reversal symmetry-breaking magnetic order lies on top of the nuclear Bragg peaks and so preserves translational symmetry, thereby excluding likely antiferromagnetic orderings of the $\mathrm{Cu}$ ions. No scattering is observed when the in-plane component of the scattering vector is zero, however, indicating, apparently inconsistently, zero net ferromagnetic moment. An interpretation of the scattering in terms of magnetic dipole moments requires that any possible magnetic dipole moments be canted at $45^{\circ}$ to the $\mathrm{Cu}-\mathrm{O}$ plane, with a magnitude of $\sim 0.1 \mu_{B}$. Some ARPES measurements also point to time-reversal symmetry breaking, with measurements on $\mathrm{Bi}_{2} \mathrm{Sr}_{2} \mathrm{CaCu}_{2} \mathrm{O}_{8-\delta}$ (Bi-2212), for example, yielding different photocurrents for left- and right-circularly polarized photons below the pseudogap onset temperature in the underdoped regime [8]. The interpretation of this result as further evidence of time-reversal symmetry breaking remains hotly disputed however (see for example Refs. [9-11]).

Apparently inconsistent with the absence of ferromagnetism indicated by the neutron data is the observation of a signal below $T^{*}$ in magneto-optic Kerr effect measurements on YBCO [12]. While such a Kerr signal provides additional confirmation of time-reversal symmetry breaking, it is usually indicative of ferromagnetic ordering which the neutron measurements exclude. The signal strength, $\sim 1 \mu \mathrm{rad}$, is about four orders of magnitude smaller than the response in a typical ferromagnetic transition-metal oxide. In addition, it shows an unusual temperature dependence, which implies that the source of the Kerr rotation is not the primary order parameter for the phase transition. Intriguingly, field-training data suggest that time-reversal symmetry is already broken above $T^{*}$ by an order parameter that interacts with that of the pseudogap phase, even though the Kerr rotation is below the detection limit in that temperature range.

Results from $\mu$ SR spectroscopy, which is sensitive to local magnetic fields at the site of muon implantation in a sample, are also contradictory. Early $\mu \mathrm{SR}$ experiments on YBCO [7], and subsequent work on $\mathrm{La}_{2-x} \mathrm{Sr}_{x} \mathrm{CuO}$ (LSCO) [13] gave upper bounds on internal static magnetic fields of 0.01 and $0.02 \mathrm{mT}$ 
respectively; these are three orders of magnitude smaller than the fields that should be present if the spin-polarized neutron scattering were caused by a magnetic dipolar ordering. It was pointed out, however, that any order fluctuating with a time scale faster than $10^{-6} \mathrm{~s}$ would be averaged to zero in a $\mu \mathrm{SR}$ experiment [13]. Later experiments on YBCO [14] even suggested an impurity phase origin for the weak magnetic signal. The small internal magnetic fields obtained in the muon measurements are supported by NMR [15] and NQR [5] studies on underdoped YBCO, which place upper limits of 0.15 and $0.07 \mathrm{mT}$ on the static local magnetic fields at the $\mathrm{Y}$ and $\mathrm{Ba}$ sites, respectively, and of $0.7 \mathrm{mT}$ at both sites for rapidly (faster than $10^{-11} \mathrm{~s}$ ) fluctuating fields.

An important theoretical description of the pseudogap phase, which is both conceptually appealing and consistent with many of the experimental observations, is the so-called orbital-current model [16]. In this model, oppositely oriented electron-current loops flow identically within each unit cell producing intra-unit-cell antiferromagnetically aligned magnetic moments. The current loops introduce magnetic moments that can be arbitrarily small depending on the magnitudes of the currents, and the ordering breaks time-reversal symmetry while retaining translational invariance. In its existing form, however, the model is seemingly inconsistent with the NMR, NQR, and $\mu$ SR results $[5,14,15]$, and a microscopic origin of the behavior is not obvious.

A recent theoretical analysis demonstrating that neutrons are deflected by magnetoelectric multipoles [17] led to the intriguing suggestion that a ferroic ordering of magnetic quadrupoles on the $\mathrm{Cu}$ ions in YBCO could have a symmetry consistent with that inferred from the neutron scattering measurements [18]. The magnetoelectric multipoles, of which the magnetic quadrupoles are one type, form the second-order term in the multipole expansion of the interaction energy of the magnetization density with a magnetic field [19] (the magnetic dipole forms the first-order term) and so break time-reversal symmetry without carrying a magnetic dipole moment. Reference [18] discounted evidence from neutron scattering, specifically the observed structure factor of the $L=0$ Bragg peak, which requires canted moments when interpreted in terms of magnetic dipole moments [20]. As a result, their proposed ordering of $z^{2}$ quadrupoles, which leads to a $C m^{\prime} m^{\prime} m^{\prime}$ space group, is inconsistent with experiment. The concept remains relevant, however, for the lower $C 2 / \mathrm{m}^{\prime}$ symmetry that captures all of the neutron data. Such a "ferromagnetoelectric" order parameter is also supported by a symmetry analysis motivated by the Kerr effect results [21], which identified magnetic point-group symmetries $2 / \mathrm{m}^{\prime}$, $m^{\prime} m^{\prime} m^{\prime}, 2 m^{\prime} m^{\prime}$ that could generate polarization rotation without a net ferromagnetism via the magnetoelectric effect. How to achieve such a ferromagnetoelectric order microscopically, however, in the absence of an additional magnetic dipolar order is far from obvious.

Here, we use first-principles calculations based on density functional theory to demonstrate the existence and calculate the magnitudes of magnetoelectric multipoles in the prototypical cuprate superconductor $\mathrm{HgBa}_{2} \mathrm{CuO}_{4+\delta}$. We show that all existing experimental reports of the pseudogap phase are consistent, to our knowledge, with its order parameter being the ferroic ordering of such magnetoelectric multipoles. We provide a mechanism for the ordering of the magnetoelectric multipoles in the absence of magnetic dipolar ordering that is mediated by coupling between fluctuating spin dipole moments and optical phonons. We show that our analysis embraces many aspects of, and provides a microscopic justification for, the theoretical models of Refs. [16,18] while also accounting for the experimental observations that they do not capture. Finally, we present proposals for experiments that could directly verify or disprove our proposed mechanism.

\section{MERCURY BARIUM COPPER OXIDE}

We choose as the subject of our study the model high- $T_{c}$ cuprate $\mathrm{HgBa}_{2} \mathrm{CuO}_{4}(\mathrm{Hg}$-1201) [22], which shows the usual cuprate phase diagram, with its characteristic superconducting dome [23] as a function of hole doping. It has a simple tetragonal $P 4 / \mathrm{mmm}$ crystal structure [Fig. 1(a)] which, importantly for our study, contains a single $\mathrm{Cu}-\mathrm{O}$ plane per unit cell, minimizing the possible magnetic ordering combinations that preserve translational symmetry. In particular, since it contains only one $\mathrm{Cu}$ ion per unit cell, any antiferromagnetic ordering of magnetic moments on the $\mathrm{Cu}$ ions is excluded. The ideal parent compound, with its +2 oxidation state for $\mathrm{Cu}$, has to our knowledge not been achieved, since there is always a nonzero amount of hole doping through incorporation of interstitial oxygen. The oxygen interstitial site is in the $\mathrm{Hg}$ plane, equidistant from the $\mathrm{Hg}$ ions, and so doping does not introduce disorder into the $\mathrm{Cu}-\mathrm{O}$ planes responsible for the superconductivity [24]. A range of hole concentrations from $\delta=0.05-0.25$ (in units of number of holes per $\mathrm{Cu}$ ion) has been reported, with a $T_{c}$ of $97 \mathrm{~K}$ at optimal doping $(\delta=0.15)$. (a)

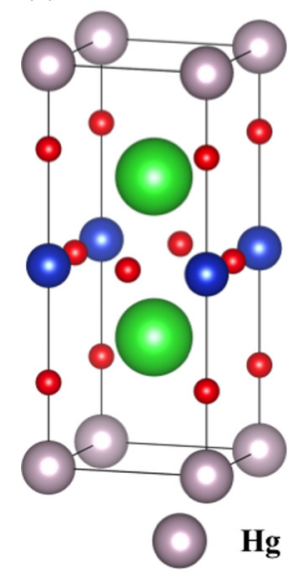

(b)

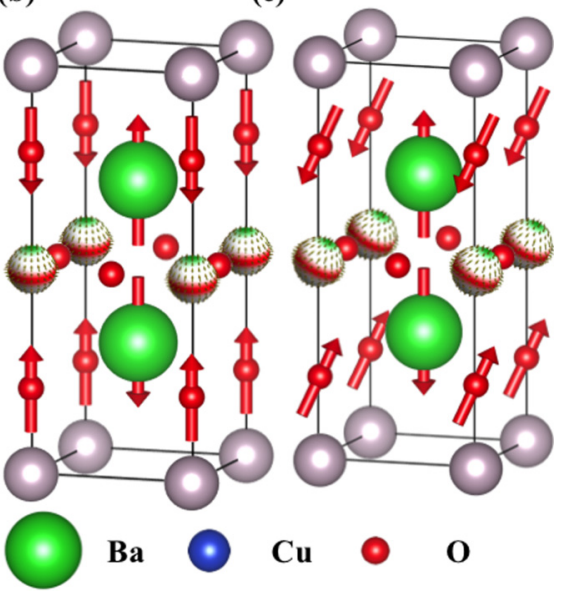

FIG. 1. (a) Structure of $\mathrm{HgBa}_{2} \mathrm{CuO}_{4}$, with $\mathrm{Cu}, \mathrm{O}, \mathrm{Ba}, \mathrm{Hg}$ represented by blue, red, green, and white spheres, respectively. (b) Allowed dipolar magnetic order for $\mathrm{HgBa}_{2} \mathrm{CuO}_{4}$ within the $\mathrm{Cm}^{\prime} m^{\prime} m^{\prime}$ magnetic space group. The red arrows indicate the atomic-site magnetic dipole moments which must be oriented along the $z$ axis. This pattern results in a unit-cell $M_{z^{2}}$ magnetic quadrupole as well as an atomic-site quadrupole on the $\mathrm{Cu}$ site as shown. (c) Possible dipolar magnetic ordering in the $C 2 / \mathrm{m}^{\prime}$ space group. The dipole moments on the apical oxygens exhibit an additional canting with respect to $C m^{\prime} m^{\prime} m^{\prime}$. This pattern results in a combination of $M_{z^{2}}$ and $M_{x z} / M_{y z}$ quadrupoles on the $\mathrm{Cu}$ site. 
The existence and doping dependence of the pseudogap phase in $\mathrm{Hg}-1201$ is confirmed from thermoelectric power measurements [23], ${ }^{63} \mathrm{Cu}[25]$ and ${ }^{17} \mathrm{O}$ [26] NMR, photoemission [27], and spin-polarized neutron diffraction $[28,29]$ to have the same overall behavior as in the other cuprate superconductors. As mentioned above, the neutron data for all cuprates point to an ordering in the pseudogap phase that breaks time-reversal symmetry but not the translational symmetry of the lattice; in the case of $\mathrm{Hg}$-1201 with one $\mathrm{Cu}$ ion per unit cell this fact, combined with the absence of ferromagnetism, excludes the presence of ordered magnetic dipole moments on the $\mathrm{Cu}$ ions. A recent meta-analysis of all available neutron studies on $\mathrm{Hg}-1201$ [30] concluded that the neutron data are consistent with the $C m^{\prime} m^{\prime} m^{\prime}$ magnetic space group which prohibits ordered magnetic dipole moments at the $\mathrm{Cu}$ sites and also breaks space-inversion symmetry. Note that experimental information for the magnetic structure factor of the $L=0$ peak is not yet available, due to the strong scattering from the nuclear $L=0$ peak [31], so it is not possible to know whether the actual magnetic symmetry is lowered to $C 2 / m^{\prime}$ (which anyway also breaks space-inversion symmetry and prohibits ordered magnetic dipoles on the $\mathrm{Cu}$ sites) as in the YBCO case. Also relevant for our discussion is the finding, using inelastic neutron scattering [29] and from interpretation of optical scattering [32], of the onset at $T^{*}$ of a weakly dispersive collective spin excitation at around $40 \mathrm{meV}$. We mention finally that charge density wave correlations have been measured using $\mathrm{Cu} L_{3}$-edge resonant x-ray scattering at a lower temperature than $T^{*}$ [33] and interpreted in terms of the buildup of significant dynamic antiferromagnetic correlations at $T^{*}$.

While the detailed analysis we present here, particularly the numerical density functional theory study, is specific to $\mathrm{Hg}$ 1201 , we believe that our conclusions are generally applicable to the whole family of high- $T_{c}$ cuprates.

\section{MAGNETOELECTRIC MULTIPOLES IN Hg-1201}

The magnetoelectric multipoles form the second-order contributions to the multipole expansion of the energy of a general magnetization density interacting with a general magnetic field [19]. (The first-order contribution comes from the magnetic dipole moment, and a true magnetic monopole would give a zeroth-order contribution.) Their operators are formed from a product of a position operator $\hat{\boldsymbol{r}}$ and a magnetization operator $\hat{\mu}$ and therefore they are only nonzero in materials that have both broken time-reversal and space-inversion symmetry. As a result, materials with nonzero magnetoelectric multipoles also exhibit a linear magnetoelectric response in which an electric field induces a proportional magnetization and vice versa. In most magnetoelectrics discussed to date, the source of the magnetoelectric multipolar ordering is an antiferromagnetic ordering of magnetic dipoles which breaks simultaneously both time-reversal and space-inversion symmetries, although inversion symmetry can also be broken separately, for example, by a polar structural distortion.

The chemical unit cell of $\mathrm{Hg}-1201$ shown in Fig. 1(a) is clearly centrosymmetric with an inversion center at the $\mathrm{Cu}$ site. In the absence of ordered magnetic moments, it is also time-reversal symmetric, so all magnetoelectric
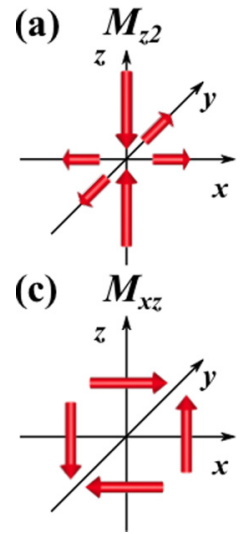
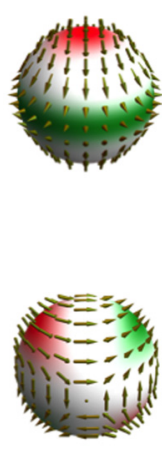

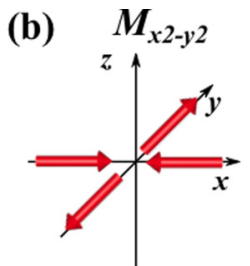

(d)
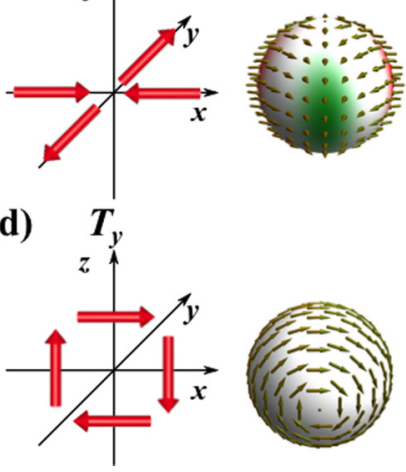

$T_{y}$

FIG. 2. Pattern of local magnetic dipole moments $\mu$ that generate magnetoelectric multipoles. (a)-(c) Show the cases of magnetic quadrupoles with $M_{z^{2}}, M_{x^{2}-y^{2}}$, and $M_{z^{2}}$ symmetries, respectively. (d) Pattern of local magnetic dipole moments that generates a toroidal moment $\boldsymbol{T}_{y}$.

multipoles at all atoms are zero. Here, we explore scenarios in which nonzero magnetoelectric multipoles can emerge from such a high-temperature centrosymmetric paramagnetic state and eventually represent a primary order parameter for a symmetry-lowering phase transition. For conciseness, we focus particularly on the magnetic quadrupole which is a second-rank tensor given by [19]

$$
M_{i j}=\frac{1}{2} \int\left[r_{i} \mu_{j}+r_{j} \mu_{i}-\frac{2}{3} \boldsymbol{r} \cdot \boldsymbol{\mu}(\boldsymbol{r}) \delta_{i, j}\right] d^{3} r,
$$

with energy of interaction $E_{\text {int }}$, with a magnetic field $\boldsymbol{H}$ determined by the field gradients according to

$$
E_{\text {int }}=-M_{i j}\left(\partial_{i} H_{j}+\partial_{j} H_{i}\right)_{r=0} .
$$

Such a magnetic quadrupole can be generated by a pattern of local magnetic dipole moments, such as that shown in Fig. 2(a), which might represent spin moments on atoms within a unit cell. The pattern in Fig. 2(a) shows a $z^{2}$ magnetic quadrupole $M_{z^{2}}$, whose magnitude is straightforwardly obtained from Eq. (1) by summing over the atoms and replacing $\boldsymbol{\mu}(\boldsymbol{r})$ by the local moments $\mu$; the values per unit cell are converted to a macroscopic "quadrupolization" by dividing by the unit-cell volume [19]. Note that the pattern of ordered dipole moments breaks the inversion symmetry. In addition to this "unit-cell" contribution to the total quadrupolization formed from the separation of magnetic dipoles at the unit-cell length scale, there is also an "atomic-site" contribution to the total quadrupolization, also illustrated in Fig. 2(a), which arises from the magnetization texture within a sphere centered at individual atoms, provided that their site symmetry is appropriate. Figure 2(b) shows the analogous unit-cell and atomic-site contributions to the $x^{2}-y^{2}$ magnetic quadrupole $M_{x^{2}-y^{2}}$, and Fig. 2(c) the $x z$ magnetic quadrupole $M_{x z}$. In Table I we list the point-group symmetry on the Cu site caused by the occurrence of these quadrupoles either individually or in various combinations on the $\mathrm{Cu}$ ion. We see that both $M_{z^{2}}$ and $M_{x^{2}-y^{2}}$ have $m^{\prime} m^{\prime} m^{\prime}$ symmetry, and any combination of either the $z^{2}$ or $x^{2}-y^{2}$ quadrupole with either the $x z$ or $y z$ quadrupole yields $2 / m^{\prime}$ symmetry. Symmetry analysis of 
TABLE I. Magnetic quadrupole tensor elements $M_{i j}$, the phonon mode symmetries by which they are induced, the magnetic point groups they create if situated on the $\mathrm{Cu}$ ion in $\mathrm{Hg}-1201$, and the nonzero elements of the corresponding magnetoelectric (ME) tensors. In the lower part, we list equal and unequal $(\chi \neq 1)$ superpositions of quadrupoles and the corresponding symmetries.

\begin{tabular}{|c|c|c|c|c|c|}
\hline$M_{i j}$ & Phonon & Point group & \multicolumn{3}{|c|}{ ME tensor } \\
\hline$z^{2}, x^{2}-y^{2}$ & $A_{2 u}, B_{2 u}$ & $m^{\prime} m^{\prime} m^{\prime}$ & {$\left[\alpha_{11}\right.$} & $\alpha_{22}$ & $\begin{array}{c}\alpha_{33} \\
\end{array}$ \\
\hline$x z / y z$ & $\mathrm{E}_{\mathrm{u}}$ & $m^{\prime} m m$ & & & $\left.k_{23}\right]$ \\
\hline$z^{2} /\left(x^{2}-y^{2}\right)+x z / y z$ & $\mathrm{~A}_{2 \mathrm{u}} / \mathrm{B}_{2 \mathrm{u}}+\mathrm{E}_{\mathrm{u}}$ & $2 / m^{\prime}$ & & $\alpha_{22}$ & $\left.\begin{array}{l}\alpha_{23} \\
\alpha_{33}\end{array}\right]$ \\
\hline$x z+\chi y z$ & $\mathrm{E}_{\mathrm{u}}$ & $2^{\prime} / m$ & $\alpha_{21}$ & $\begin{array}{l}\alpha_{12} \\
\alpha_{32}\end{array}$ & $\alpha_{23}$ \\
\hline$x z+\chi y z+z^{2}$ & $\mathrm{E}_{\mathrm{u}}+\mathrm{A}_{2 \mathrm{u}}$ & $-1^{\prime}$ & {$\left[\begin{array}{l}\alpha_{11} \\
\alpha_{21} \\
\alpha_{31}\end{array}\right.$} & $\begin{array}{l}\alpha_{12} \\
\alpha_{22} \\
\alpha_{32}\end{array}$ & $\left.\begin{array}{l}\alpha_{13} \\
\alpha_{23} \\
\alpha_{33}\end{array}\right]$ \\
\hline
\end{tabular}

the toroidal magnetoelectric multipoles shows that a toroidal moment oriented in the $y$ direction $\boldsymbol{T}_{y}$ on the $\mathrm{Cu}$ site has the same $m^{\prime} m m$ symmetry as $M_{x z}$ and $M_{y z}$, so its combination with the $z^{2}$ or $x^{2}-y^{2}$ quadrupole also yields the proposed $2 / m^{\prime}$ symmetry. We illustrate such a $\boldsymbol{T}_{y}$ toroidal moment in Fig. 2(d). (Note that the toroidal moments are sometimes called anapoles in the literature.) The magnetoelectric monopole has the same $m^{\prime} m^{\prime} m^{\prime}$ symmetry as the $z^{2}$ quadrupole and so is always simultaneously allowed. Since magnetoelectric monopoles have been shown to not interact with neutrons, however [17], we do not discuss them here.

In the remainder of this section, we discuss three possible mechanisms that could lead to the formation of magnetoelectric multipoles in $\mathrm{HgBa}_{2} \mathrm{CuO}_{4+\delta}$. First, we analyze the possibility that they are generated by only ordering of static spin-dipole moments as has been previously discussed in the analysis of neutron scattering data. Second, we revisit the leading orbital-current model in the context of magnetoelectric (ME) multipoles. And, finally, we suggest a new mechanism in which a coupling between fluctuating spin dipoles and phonons can generate a quasistatic ordering of the ME multipoles.

\section{A. Possibilities for generating magnetoelectric multipoles from magnetic dipolar ordering}

First, we analyze the possibilities within the highersymmetry $C m^{\prime} m^{\prime} m^{\prime}[18]$ and possible lower-symmetry $C 2 / m^{\prime}$ space groups of generating a magnetic quadrupole at the $\mathrm{Cu}$ site in the $\mathrm{HgBa}_{2} \mathrm{CuO}_{4}$ unit cell through an ordered arrangement of magnetic dipoles, noting that the arrangement must be antiferromagnetic with no overall magnetization. Within the $C m^{\prime} m^{\prime} m^{\prime}$ magnetic space group, magnetic dipole moments on atomic sites can be nonzero only on the apical oxygen or barium atoms, and in both cases must be aligned along the tetragonal $c$ axis [Fig. 1(b)]. As mentioned above, symmetry analysis (see Table I) shows that the $m^{\prime} m^{\prime} m^{\prime}$ pointgroup symmetry at the $\mathrm{Cu}$ site permits either an $M_{z^{2}}$ or $M_{x^{2}-y^{2}}$ quadrupole on the $\mathrm{Cu}$ ion but no magnetic dipole moment. We find that $M_{x^{2}-y^{2}}$ quadrupoles can not be induced from combinations of symmetry-allowed magnetic dipole moments if the moments are restricted to lie on the atomic sites. Static magnetic moments on $\mathrm{Ba}$ and/or $\mathrm{O}$ aligned along the $c$ axis, on the other hand, do create a $M_{z^{2}}$ quadrupole on $\mathrm{Cu}$, as illustrated in Fig. 1(b).

We emphasize, however, that the presence of a magnetic moment on either oxygen or barium is unlikely, particularly in the absence of magnetic moments on $\mathrm{Cu}$, due to the closed-shell configurations of both ions in their formal charge state. To investigate the energetics, we performed a constrained-moment density functional calculation with the oxygen magnetic moments set to the pattern of Fig. 1(b) and the magnitude of $0.1 \mu_{B}$ suggested by the neutron data, and find an energy cost of $\sim 100 \mathrm{meV}$ per formula unit over the nonmagnetic case. We recall, in addition, that such large magnetic dipole moments are excluded by the absence of internal magnetic fields indicated by NMR measurements, which in the case of $\mathrm{HgBa}_{2} \mathrm{CuO}_{4}$ have an upper bound of $0.1 \mathrm{mT}[26]$.

The lower-symmetry $C 2 / m^{\prime}$ space group can be reached by canting of the oxygen magnetic moments away from the $z$ axis occurs, as illustrated in Fig. 1(c). The magnetoelectric multipole illustrated on the $\mathrm{Cu}$ atoms in Fig. 1(c) is the equal linear combination of the $M_{z^{2}}$ and $M_{x z}$ quadrupoles; other combinations as listed in Table I are allowed by symmetry. The difficulties discussed above, i.e., that creating dipole moments on $\mathrm{O}$ in the absence of ordered moments on $\mathrm{Cu}$ is energetically expensive and that the absence of internal fields in the NMR data permits only very small values of magnetic dipole moments, persist for this site symmetry.

\section{B. Magnetoelectric multipoles from orbital currents}

In the orbital-current mechanism [16], the symmetry considerations discussed above are less restrictive since the magnetic dipole moments generated by the currents are not required to reside on atoms. First, we note that the original proposal of currents orbiting within the $\mathrm{Cu}-\mathrm{O}$ plane (the socalled $\theta_{2}$ phase) [16], which would generate dipole moments parallel to the $c$ axis, is inconsistent with $C m^{\prime} m^{\prime} m^{\prime}$ or $C 2 / m^{\prime}$ symmetry, which forbids dipole moments parallel to the $c$ axis within the $\mathrm{Cu}-\mathrm{O}$ plane. In fact, the $\theta_{2}$ phase can be considered to be a combination of a $\boldsymbol{T}_{y}$ toroidal moment with an $x z$ magnetic quadrupole [34], which we see from Table I has $\mathrm{m}^{\prime} \mathrm{mm}$ symmetry. Reference [35] contains a comprehensive discussion of modified orbital-current models and proposes one pattern Fig. 2(d) of Ref. [35]) that is consistent with all of the latest neutron and Kerr effect data. This orbital-current pattern is consistent with our Fig. 1(c) and corresponds to a combination of $M_{z^{2}}$ and $M_{x z}$ quadrupoles. Indeed, there is a clear correspondence between magnetoelectric multipoles and orbital currents, with the important difference that the orbital currents generate their multipolar character from combinations of magnetic dipoles. We return later to a discussion of the consequences of this difference in microscopic origin, and in particular how it could be used to distinguish the microscopic mechanisms experimentally. 
(a)

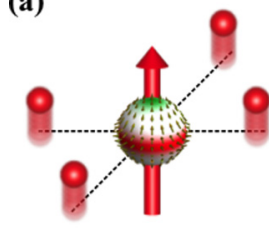

(d)
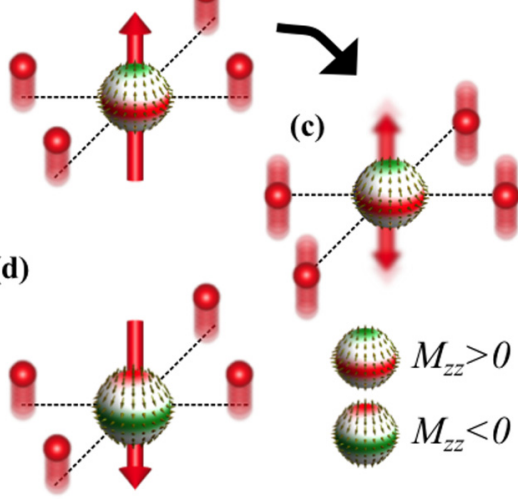

(e)

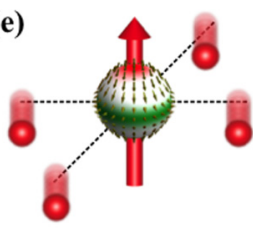

FIG. 3. (a)-(e) Schematics illustrating the formation of a quasistatic magnetic quadrupole via the spin-phonon coupling mechanism. The red spheres represent oxygen ions, which form a squareplanar coordination around the central $\mathrm{Cu}$ ion. A polar distortion as shown in (a) generates a magnetic quadrupole moment on the central $\mathrm{Cu}$ ion because of its magnetic dipole moment. The magnitude of the magnetic quadrupole moment is determined by the product of the size of the distortion and the size of the dipole moment; its sign is determined by the direction of the distortion and the orientation of the magnetic dipole moment. Therefore, when the orientation of the $\mathrm{Cu}$ magnetic moment changes (d) without a change in the direction of distortion, the opposite magnetic quadrupole moment is generated; likewise, when the direction of distortion changes (e) without a change in the magnetic moment orientation, the magnetic quadrupole reverses. If the spin-phonon coupling correlates the orientation of the magnetic dipole moment with the direction of the polar distortion so that both change simultaneously (b), then the sign of the magnetoelectric moment is unchanged (c).

\section{Dynamically ordered magnetoelectric multipoles from spin-phonon coupling}

Finally, we suggest a mechanism in which a spontaneous ferroic ordering of magnetic quadrupoles could emerge from a dynamically fluctuating system of paramagnetic spin dipole moments on the $\mathrm{Cu}$ ions through coupling between the spins and an optical phonon. Within our model, the space-inversion symmetry at the $\mathrm{Cu}$ site is broken due to excitation of an optical phonon which shifts the $\mathrm{Cu}$ ion from the center of its oxygen coordination plane. Since the $\mathrm{Cu}$ ion carries a local (albeit fluctuating) magnetic dipole moment, it therefore acquires a magnetoelectric multipole (again fluctuating) when the space-inversion symmetry is broken. We illustrate this in Fig. 3 for the case of the $z^{2}$ magnetic quadrupole coupled to a phonon of $A_{2 u}$ symmetry, noting that the mechanism is general, with different magnetoelectric multipoles coupling to different optical phonons as listed in Table I.

For a specific direction of off centering of the $\mathrm{Cu}$ ion relative to the oxygen plane, a reversal of the magnetic dipole moment, such as occurs through thermal fluctuation, simultaneously reverses the magnetic quadrupole [Figs. 3(a) and 3(b)]. Therefore, as expected, a straightforward Langevin disordered-local-moment paramagnet also has no net ordering of its magnetic quadrupoles. Note, however, that a reversal of the direction of displacement of the $\mathrm{Cu}$ ion relative to the oxygen ions, without a reversal of the magnetic dipole moment, also reverses the local $\mathrm{Cu}$ quadrupole [Fig. 3(e)]. Therefore, if a coupling between the local moment and the optical phonon exists, such that a reversal of the local dipole moment is accompanied by a reversal of the $\mathrm{Cu}$ ionic displacement, the net quadrupole moment is nonzero. Within this picture, while the time averages of both the magnetic dipole moment $\langle\boldsymbol{\mu}\rangle_{T}$ and the atomic displacements $\langle\boldsymbol{r}\rangle_{T}$ are zero, the time-averaged expectation values of the form $\langle\boldsymbol{\mu} \cdot \boldsymbol{r}\rangle_{T}$, in particular that of the magnetic quadrupole, are nonzero [Fig. 3(c)].

The magnitude of this quasistatic quadrupole within the unit cell is determined by the distance that the $\mathrm{Cu}$ ion is displaced from its centrosymmetric position $r_{z}$, and is given by $M_{z^{2}}=\frac{2}{3} r_{z} \mu_{z}$, where $\mu_{z}$ is the magnitude of the fluctuating magnetic dipole moment on the $\mathrm{Cu}$ ion $\left(\sim 1 \mu_{B}\right.$ for $\left.d^{9} \mathrm{Cu}\right)$. For a $\mathrm{Cu}$ displacement of $5 \mathrm{pm}$, which might be expected in a thermally activated phonon at the pseudogap ordering temperature, the magnitude of the unit-cell quadrupolization is around two orders of magnitude smaller than that of the prototypical magnetoelectric $\mathrm{Cr}_{2} \mathrm{O}_{3}$. In addition, the $\mathrm{Cu}$ ion develops a local onsite quadrupole due to the local asymmetry in its magnetization density as discussed above; we will calculate its magnitude in the next section.

\section{COMPUTATIONAL DETAILS}

Our electronic structure calculations are based on density functional theory within the local spin density approximation (LSDA). To account for the strong electron-electron interactions on the $\mathrm{Cu} d$ orbitals, we incorporate the widely used Hubbard $U$ and $J$ corrections of 8 and $1 \mathrm{eV}$, respectively [36-39], within the LSDA $+U$ method, with double counting treated within the fully localized limit. Structural parameters, phonon frequencies, and spin-phonon coupling constants are calculated using the Vienna $a b$ initio simulation package (VASP) [40] within the projector augmented wave (PAW) method [41] using default VASP PAW pseudopotentials with the following electrons in the valence: $\mathrm{Hg}\left(5 d^{10} 6 s^{2}\right), \mathrm{Ba}$ $\left(5 s^{2} 5 p^{6} 6 s^{2}\right), \mathrm{Cu}\left(3 p^{6} 4 s^{1} 3 d^{10}\right)$, and $\mathrm{O}\left(2 s^{2} 3 p^{4}\right)$. Convergence of forces to $0.01 \mathrm{meV} / \AA$ are obtained with a $17 \times$ $17 \times 11 k$-point mesh in combination with a cutoff energy of $600 \mathrm{eV}$.

We calculate the atomic-site magnetoelectric multipoles via a generalized density matrix decomposition using our recent implementation [19] within the linearized augmented plane wave (LAPW) ELK code [42]. The unit-cell quadrupoles are calculated according to Eq. (1) by summation over local moments, taken to be the projected magnetic dipole moments in each muffin-tin sphere. As numerical parameters for the LAPW calculations we use a basis set of $l_{\max (\mathrm{apw})}=12$, the same $k$-point sampling of the Brillouin zone as in the VASP calculations, and we take the product of the muffintin radius $(1.2,1.5,1.1$, and $0.7 \AA$ for $\mathrm{Hg}, \mathrm{Ba}, \mathrm{Cu}$, and $\mathrm{O}$, respectively) and the maximum reciprocal lattice vector to be 7.5. The total energy for both codes is converged to within $1 \mu \mathrm{eV}$ using these settings. To introduce hole doping, we reduce the total electron count in our selfconsistent calculation by the corresponding amount, while adding a uniform background charge to maintain charge neutrality, as used previously in Refs. [43-47] to calculate 
various doping-dependent properties of the $\mathrm{HgBa}_{2} \mathrm{CuO}_{4+\delta}$ family.

\section{DENSITY FUNCTIONAL CALCULATIONS OF SPIN-PHONON COUPLING AND MAGNETOELECTRIC MULTIPOLES IN HG-1201}

We begin by calculating the structure of $\delta=0.05$ doped $\mathrm{Hg}-1201$ without allowing for spin polarization to obtain a paramagnetic reference state. Our resulting structure (Table II) agrees well with the experimentally reported structure (Ref. [24]) while showing the usual LDA underestimation of the unit-cell volume (3.8\% in this case). Using our calculated structure, we then compute the total energies and phonon spectra, via the frozen phonon method, for ferromagnetic and checkerboard antiferromagnetic (in a $\sqrt{2} \times \sqrt{2} \times 1$ supercell) orderings.

The nearest-neighbor Heisenberg exchange constant $J$ is then obtained directly from the energy difference between the ferromagnetically (FM) ordered and in-plane checkerboard antiferromagnetically (AFM) ordered magnetic moment arrangements within the same crystal structure $J=-\frac{1}{2}\left(E_{\mathrm{AFM}}-\right.$ $\left.E_{\mathrm{FM}}\right)$. We obtain $J=78 \mathrm{meV}$, consistent with literature values [48]. Here, $E_{\mathrm{AFM}}$ is the energy of the $\sqrt{2} \times \sqrt{2} \times 1$ AFM-ordered unit cell containing two formula units, and $E_{\mathrm{FM}}$ is the corresponding $\mathrm{FM}$ energy. We take as phonon frequencies $\omega_{i}=\sqrt{\frac{\omega_{\mathrm{FM}_{i}}^{2}+\omega_{\mathrm{AFM}_{i}}^{2}}{2}}$, where $\omega_{\mathrm{AFM}_{i}}$ and $\omega_{\mathrm{FM}_{i}}$ are the frequencies of mode $i$ for the AFM and FM magnetic orderings, respectively. In Table III, we compare our calculated phonon frequencies with available experimental data $[49,50]$ and obtain excellent agreement, with the largest deviation from experiment being only $6 \%$.

The lowest-order spin-phonon coupling between a polar phonon and a parity-even spin arrangement is quadratic in both spin and phonon amplitude, therefore, we extract the spin-phonon coupling constants as

$$
g_{i}=\frac{\omega_{\mathrm{FM}_{i}}^{2}-\omega_{\mathrm{AFM}_{i}}^{2}}{4 S_{l}^{2}},
$$

where $S_{l}$ is the size of the local spin dipole moment. We find that, of the nine nontranslational polar modes, only seven have a sizable frequency difference between the FM and AFM

TABLE II. Experimental (Expt) [24] and calculated in this work (DFT) lattice constants and atomic positions for $\mathrm{HgBa}_{2} \mathrm{CuO}_{4}$. The Wyckoff positions are given according to the $C m^{\prime} m^{\prime} m^{\prime}$ magnetic space group; 41 and $4 \mathrm{k}$ are the only internal degrees of freedom.

\begin{tabular}{lccc}
\hline \hline Lattice constant & & DFT & Expt \\
\hline $\mathrm{a}(\AA)$ & 3.84 & 3.87 \\
$\mathrm{c} / \mathrm{a}$ & & 2.44 & 2.45 \\
Atom & Wyckoff position & DFT & Expt \\
\hline $\mathrm{Hg}$ & $2 \mathrm{a}$ & 0.00 & 0.00 \\
$\mathrm{Ba}$ & $4 \mathrm{l}$ & 0.30 & 0.30 \\
$\mathrm{Cu}$ & $2 \mathrm{~b}$ & 0.50 & 0.50 \\
$\mathrm{O}_{1}$ & $4 \mathrm{f}$ & 0.50 & 0.50 \\
$\mathrm{O}_{2}$ & $4 \mathrm{k}$ & 0.21 & 0.21 \\
\hline \hline
\end{tabular}

TABLE III. Comparison of experimental (Expt) and calculated in this work (DFT) phonon frequencies at the zone center $(q=0)$ of $\mathrm{Hg}-1201$. The values marked with an asterisk $\left(^{*}\right)$ were obtained from a shell-model calculation and an interpolation to the zone center.

\begin{tabular}{lrcr}
\hline \hline $\begin{array}{l}\text { Mode } \\
\text { symmetry }\end{array}$ & DFT & $\begin{array}{c}\text { Frequency, } \omega(\mathrm{meV}) \\
\text { Expt [50] }\end{array}$ & Expt [49] \\
\hline $\mathrm{E}_{\mathrm{u}}$ & 7.7 & $7.24^{*}$ & \\
$\mathrm{E}_{\mathrm{g}}$ & 9.3 & 9.43 & \\
$\mathrm{~A}_{2 \mathrm{u}}$ & 10.3 & & \\
$\mathrm{E}_{\mathrm{u}}$ & 19.0 & $19.9^{*}$ & \\
$\mathrm{~A}_{2 \mathrm{u}}$ & 19.4 & & 20.0 \\
$\mathrm{~A}_{1 \mathrm{~g}}$ & 20.2 & $20.1^{*}$ & \\
$\mathrm{E}_{\mathrm{g}}$ & 21.2 & & \\
$\mathrm{E}_{\mathrm{u}}$ & 28.9 & $29.5^{*}$ & \\
$\mathrm{~B}_{2 \mathrm{u}}$ & 32.0 & & \\
$\mathrm{E}_{\mathrm{u}}$ & 42.3 & $45.4^{*}$ & \\
$\mathrm{~A}_{2 \mathrm{u}}$ & 46.3 & & 73.4 \\
$\mathrm{E}_{\mathrm{u}}$ & 68.5 & $71.8^{*}$ & \\
$\mathrm{~A}_{1 \mathrm{~g}}$ & 70.8 & $72.3^{*}$ & \\
$\mathrm{~A}_{2 \mathrm{u}}$ & 76.1 & & \\
\hline \hline
\end{tabular}

orderings, and hence a considerable spin-phonon coupling. All of these strongly spin-phonon coupled modes, which we show in Fig. 4 (note that two are twofold degenerate), correspond to relative displacements of the $\mathrm{Cu}$ cation and its coordinating oxygen anions. The frequencies of these modes span the range from $20-70 \mathrm{meV}$ and we list the corresponding couplings in Table IV. (In the following, we use as labels for each phonon mode its irreducible representation followed in brackets by its frequency in $\mathrm{meV}$ ).

Freezing in the displacement patterns of each of these polar modes using FM magnetic ordering induces an atomic-site magnetic quadrupole on the $\mathrm{Cu}$ site of symmetry determined

(a)

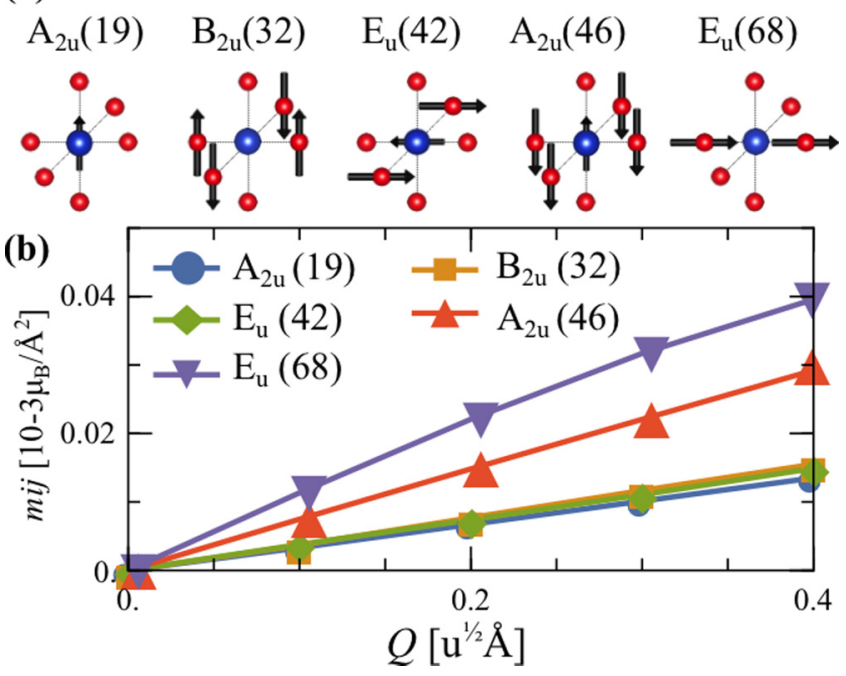

FIG. 4. (a) Schematics of $\mathrm{CuO}$ modes with the strongest spinphonon coupling. The labels above the pictures represent the mode symmetry and frequency in meV. (b) Variation of the local quadrupolar moments $m_{i j}$ as a function of phonon mode amplitudes for all modes shown schematically in (a). 
TABLE IV. Phonon frequencies, spin-phonon coupling constants, magnetic quadrupole symmetry, and atomic-site $m_{i j}$ and unit-cell $M_{i j}$ magnetic quadrupole values for quarter amplitudes $(Q=0.25)$ of selected polar modes in $\mathrm{Hg}-1201$.

\begin{tabular}{lccccc}
\hline \hline $\begin{array}{l}\text { Mode } \\
\text { symmetry }\end{array}$ & $\begin{array}{c}\omega \\
(\mathrm{meV})\end{array}$ & $\begin{array}{c}g \\
(\mathrm{meV})\end{array}$ & $\begin{array}{c}\text { Quadrupole } \\
\text { symmetry }\end{array}$ & $\begin{array}{c}m_{i j} \\
\left(10^{-3} \frac{\mu_{B}}{\AA^{2}}\right)\end{array}$ & $\begin{array}{c}M_{i j} \\
\left(10^{-3} \frac{\mu_{B}}{\AA^{2}}\right)\end{array}$ \\
\hline $\mathrm{A}_{2 \mathrm{u}}$ & 19.4 & -0.8 & $z^{2}$ & 0.01 & 0.70 \\
$\mathrm{~B}_{2 \mathrm{u}}$ & 32.0 & -8.1 & $x^{2}-y^{2}$ & 0.01 & 0 \\
$\mathrm{E}_{\mathrm{u}}$ & 42.3 & -14.7 & $x z / y z$ & 0.01 & 0.10 \\
$\mathrm{~A}_{2 \mathrm{u}}$ & 46.3 & -5.7 & $z^{2}$ & 0.02 & 0.41 \\
$\mathrm{E}_{\mathrm{u}}$ & 68.5 & -7.0 & $x z / y z$ & 0.03 & 0.00 \\
\hline \hline
\end{tabular}

by the symmetry of the phonon: $M_{z^{2}}$ for the $\mathrm{A}_{2 \mathrm{u}}$ modes, $M_{x^{2}-y^{2}}$ for the $\mathrm{B}_{2 \mathrm{u}}$ mode, and $M_{x z}$ or $M_{y z}$ for the $\mathrm{E}_{\mathrm{u}}$ modes, where for the latter case the orientation of the doubly degenerate mode determines the symmetry of the quadrupole. In Fig. 4, we show our calculated magnitudes for the $\mathrm{Cu}$ atomic-site magnetic quadrupoles, obtained from decomposition of the density matrix [19], as a function of the amplitude of the phonon eigenvector for all five independent modes. We see that the response is linear in each case, with the $\mathrm{E}_{\mathrm{u}}(68)$ and $\mathrm{A}_{2 \mathrm{u}}(46)$ modes exhibiting the largest slope. To be able to compare the induced moments, we list the atomic-site magnetic quadrupole moments $m_{i j}$ at one quarter amplitude of the normalized eigenvector $(Q=0.25)$ for each mode in Table IV. While the amplitude of the phonon excitations depends on both the frequency and the temperature, a quarter amplitude corresponds approximately to the average of their thermally excited displacements at $200 \mathrm{~K}$ and represents a maximum relative copper-oxygen displacement of $6 \mathrm{pm}$. Note that the quadrupolar moment in the undistorted structure is zero by symmetry.

Next, we calculate the induced unit-cell magnetic quadrupole $M_{i j}$ for each mode using Eq. (1), where the integral is replaced by the sum over the local dipole moments $\mu$ on the $\mathrm{Cu}$ ions and the positions of the local dipole moments are set by the mode pattern of each phonon mode. Note that the symmetries of the allowed induced unit-cell quadrupoles are the same as those of the atomic-site quadrupoles for the corresponding mode. As before, the magnitude of the induced quadrupole is linear in the amplitude of the mode; in Table IV we list the $M_{i j}$ values again for $Q=0.25$. We find the strongest unit-cell quadrupolar responses from the $\mathrm{A}_{2 \mathrm{u}}(46)$ and $\mathrm{A}_{2 \mathrm{u}}(19)$ modes, both of which exhibit a significant amount of $\mathrm{Cu}$ movement [see Fig. 3(a)]. In contrast, the asymmetric mode pattern of $\mathrm{B}_{2 \mathrm{u}}(32)$ induces no unit-cell magnetic quadrupole on symmetry grounds.

The total quadrupole moment per unit cell induced by a specific mode is the sum of atomic-site and unit-cell contributions. We find for most modes that the latter contribution is dominant and at least one order of magnitude larger than the atomic-site quadrupole. Consequently, the $\mathrm{A}_{2 \mathrm{u}}(46)$ and $\mathrm{A}_{2 \mathrm{u}}(19)$ modes induce the largest magnetic quadrupoles in $\mathrm{Hg}-1201$. Our quantitative analysis confirms our earlier estimate that the size of these magnetic quadrupoles for typical thermally activated mode amplitudes is approximately two orders of magnitude smaller than that of $\mathrm{Cr}_{2} \mathrm{O}_{3}$.
Comparison of the magnitudes of the spin-phonon coupling and the induced magnetic quadrupoles shows that the strongest spin-phonon coupling does not necessarily result in the largest magnetic quadrupoles; specifically, the strongest spin-phonon coupling is found for the $\mathrm{E}_{\mathrm{u}}(42)$ mode, whereas the $\mathrm{E}_{\mathrm{u}}(68)$ mode induces the strongest atomic-site and the $A_{2 u}(19)$ mode the strongest unit-cell magnetoelectric quadrupolar moment. The spin-phonon coupling is determined by the change in magnetic exchange interaction on changing bond angles and lengths, whereas the source of the induced unit-cell quadrupolar moment is the generated asymmetry in magnetization at the $\mathrm{Cu}$ site. The exchange interactions are dominated in turn by the $\mathrm{Cu}-\mathrm{O}-\mathrm{Cu}$ bond angle, which changes by $8.2^{\circ}$ per mode amplitude for $\mathrm{E}_{\mathrm{u}}(42)$ and only $2.8^{\circ}$ for $\mathrm{A}_{2 \mathrm{u}}(19)$. In contrast, the spin asymmetry also depends on the shift of the $\mathrm{Cu}$ ion away from its centrosymmetric position; this is $0.10 \mathrm{pm}$ per mode amplitude for $\mathrm{A}_{2 \mathrm{u}}(19)$ and only $0.04 \mathrm{pm}$ for $\mathrm{E}_{\mathrm{u}}(42)$.

\section{SPIN-PHONON COUPLING AND DYNAMICAL FERRO-ORDERING OF THE MAGNETOELECTRIC MULTIPOLES}

Finally, we present a simple "toy model" simulation of the time evolution of our coupled spin-phonon system to illustrate that, while the spin fluctuations yield an average magnetization of zero, and the phonon vibrations yield an average lattice displacement of zero, the coupling between the two yields a nonzero average magnetoelectric multipole. We use the following Hamiltonian to describe the coupling between the polar phonon modes and the spin lattice:

$$
\hat{H}=J \sum_{\langle n n\rangle} S_{l} \cdot S_{m}+\sum_{i} \frac{\omega_{i}^{2}}{2} Q_{i}^{2}+\sum_{\langle n n\rangle, i} g_{i} Q_{i}^{2} S_{l} \cdot S_{m} .
$$

Here, $\sum_{\langle n n\rangle}$ and $\sum_{i}$ indicate summation over nearest-neighbor spins and phonon modes, respectively. The first term is the usual Heisenberg spin Hamiltonian in which localized spins $S_{l}$ interact via exchange interaction $J$, and the second term is the usual harmonic oscillator, with $Q_{i}$ giving the amplitude of the (in this case polar) $i$ th phonon mode of frequency $\omega_{i}$. The last term describes the lowest-order spin-phonon coupling between polar modes and parity-even spin arrangements, with coupling constant $g_{i}$. We use the values of the magnetic exchange constant $J$, phonon frequencies $\omega_{i}$, and spin-phonon coupling constants $g_{i}$ calculated in the previous section using density functional theory for the composition $\mathrm{HgBa}_{2} \mathrm{CuO}_{4.05}$ $(\delta=0.05)$. Note that coupling to the phonon modulates the exchange interaction between the spins creating an effective time-dependent magnetic exchange constant $J_{\text {eff }}=$ $J+g_{i} Q_{i}(t)^{2}$. Likewise, coupling to the spin system modulates the phonon frequency, giving $w_{\text {eff, } i}=2 \sqrt{w_{i}^{2} / 2+g_{i} \sum S_{l} S_{m}}$.

The spin-phonon coupling contributes a force on the atoms, through $F=-d \hat{H} / d Q=\left(\omega^{2}+g \sum_{\langle n\rangle} S_{l} S_{m}\right) Q$. We treat this spin-phonon term as a driving force on the oscillator and solve the classical equation of motion

$$
Q^{\prime \prime}(t)+2 \gamma Q^{\prime}(t)+\omega_{0}^{2} Q=2 g Q S_{l m}(t),
$$

where $S_{l m}(t)=\sum_{\langle n n\rangle} S_{l}(t) S_{m}(t)$ is the time-dependent exchange sum and we include a Stokes friction term $\gamma$ to account for the finite lifetimes of oscillator modes. 
(a)

(b)

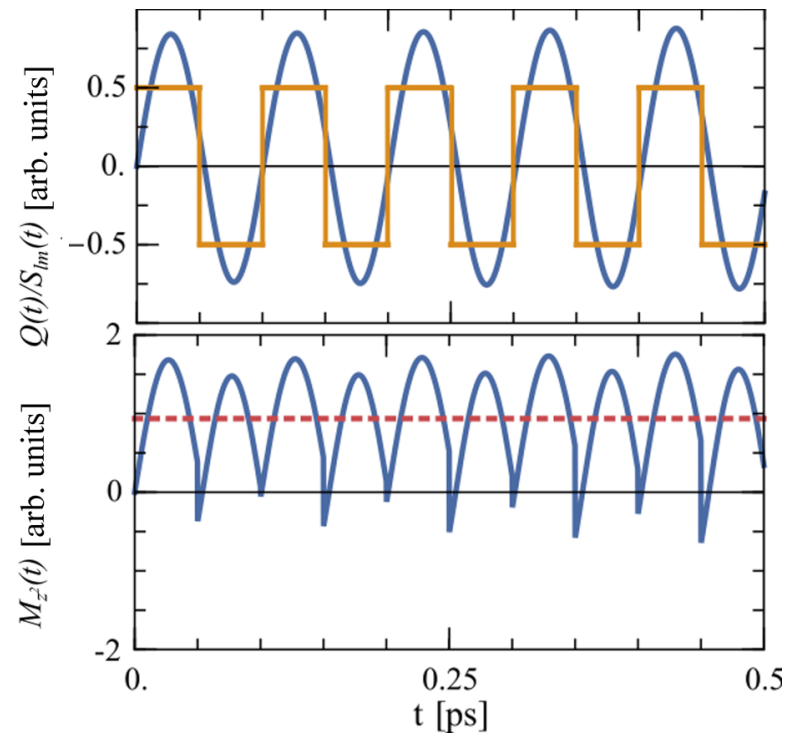

FIG. 5. (a) Phonon $Q(t)$ for the mode $\mathrm{A}_{2 \mathrm{u}}(46)$ (blue) and spin $S_{l m}(t)$ (orange) amplitude as a function of time. (b) Expectation value of the quadrupole moment $M_{z^{2}}(t)$ as a function of time. The red dotted line shows the time average.

In Figs. 5(a) and 5(b), we show our calculated time dependencies of the atomic displacements, spin expectation value, and magnetic quadrupole moment, respectively, obtained by solving Eq. (5) [51]. Our calculations were performed for parameters of the $\mathrm{A}_{2 \mathrm{u}}(46)$ mode, and with the spin term taken to be $S_{l m} \propto \operatorname{sgn}[\sin (\omega t)$ ], with the sgn function quantizing the spin fluctuations to adopt values $S_{l}= \pm \frac{1}{2}$. The spin-fluctuation frequency $\omega$ was set to $\omega_{0}=46 \mathrm{meV}$, the eigenfrequency of the $A_{2 u}$ mode. We see that, as expected, the time-averaged expectation values of both the mode amplitude and the spin expectation values, $\langle Q\rangle_{T}$ and $\left\langle S_{l m}(t)\right\rangle_{T}$, are zero, however, the expectation value of the magnetoelectric multipole $\left\langle Q \cdot S_{l m}(t)\right\rangle_{T}$ is nonzero. Consequently, a quasistatic magnetoelectric multipolar order arises from the spin-phonon coupling even in the absence of static magnetic and electric dipolar order. When we shift the frequency of the spin fluctuations away from $\omega_{0}$, the maximum and average magnetic quadrupolar amplitudes decrease as the driving term frequency becomes nonresonant with the mode.

\section{COMPARISON WITH EXPERIMENTS}

Next, we compare the behavior of our proposed quasistatic magnetoelectric quadrupolar order in Hg-1201 with experimental measurements of the behavior of the pseudogap phase. First, we note that any of the $\mathrm{A}_{2 \mathrm{u}}$ or $\mathrm{B}_{2 \mathrm{u}}$ modes, which induce $M_{z^{2}}$ or $M_{x^{2}-y^{2}}$ magnetic quadrupoles, respectively, result in the $m^{\prime} m^{\prime} m^{\prime} \mathrm{Cu}$ point-group symmetry that is consistent with existing neutron diffraction measurements. Of these, the $\mathrm{A}_{2 \mathrm{u}}(46)$ is compatible with the observed Ising-type excitation [29], and combines strong spin-phonon coupling with a sizable quadrupolar response, therefore, we restrict any mode-specific discussion to the $\mathrm{A}_{2 \mathrm{u}}(46)$ mode for conciseness. We note, however, that the model is more generally applicable, and for example the possible lower $2 / \mathrm{m}^{\prime}$ symmetry can be reached

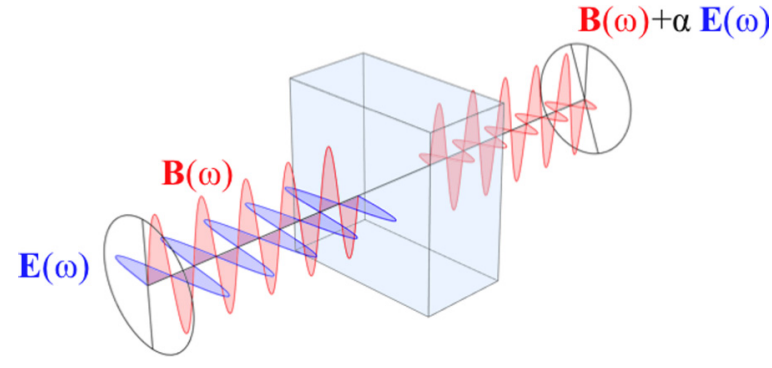

FIG. 6. Mechanism for the Kerr effect from a diagonal magnetoelectric material. The electromagnetic wave induces a magnetization parallel to its electrical component through the magnetoelectric effect. The net magnetization, and hence the plane of polarization of the light, therefore rotates relative to that of the incoming wave.

from a combination of the $\mathrm{A}_{2 \mathrm{u}}$ or $\mathrm{B}_{2 \mathrm{u}}$ modes, and the $\mathrm{E}_{\mathrm{u}}$ modes, which induce $M_{x z}$ or $M_{y z}$ quadrupoles and a $T_{y}$ toroidal moment. We note also that most high- $T_{c}$ cuprates show similar polar phonons within the copper-oxygen plane at similar frequencies, and so we do not limit our comparison to experimental data for $\mathrm{Hg}-1201$.

We begin with a discussion of the Kerr effect data. Reference [21] showed that a Kerr rotation should be induced by a magnetoelectric material with either the $m^{\prime} m^{\prime} m^{\prime}$ or $2 / m^{\prime}$ magnetic point-group symmetries that form the basis of our model. The mechanism for the Kerr rotation rests on the diagonal linear magnetoelectric response, as illustrated by the cartoon in Fig. 6. The strength of the Kerr rotation is determined in part by the strength of the magnetoelectric coupling, for which the magnetic quadrupole provides a measure in our model. Consequently, we expect from our model a Kerr rotation that is around two orders of magnitude smaller than that of $\mathrm{Cr}_{2} \mathrm{O}_{3}$, which is consistent with the findings of Refs. [12,52]. Our quasistatic magnetoelectric multipole model is therefore consistent with the observed Kerr rotation in the pseudogap phase.

Next, we discuss the local probe NMR and $\mu$ SR measurements. Both methods have been used extensively to search for static magnetic dipole moments in cuprates, motivated in large part by an attempt to verify the orbital-current model. Neither method, applied to any of the cuprates, has succeeded in finding internal magnetic fields corresponding to a static dipolar ordering, giving vanishingly small upper bounds on the possible static dipole moments $[5,13,15]$. Here, we calculate the internal fields, from point quadrupolar calculations, generated by the ferroic ordering of the local, atomic-site magnetic quadrupoles. We find that the local $M_{z^{2}}$ quadrupole on $\mathrm{Cu}$ which is induced by quarter displacement of the $\mathrm{A}_{2 \mathrm{u}}(46)$ mode generates a magnetic field of only $0.03 \mathrm{mT}$ at its neighboring oxygen sites. We note that this field strength is below the static detection limit of NMR and so is consistent with the absence of a signal in these measurements. We note also that fluctuating dipolar moments at frequencies above $0.04 \mathrm{GHz}(\mu \mathrm{SR})$ or $100 \mathrm{GHz}(\mathrm{NMR})$ could not be excluded from the resonance experiments. In our mechanism, the spin oscillates at frequencies close to the eigenfrequency of the $\mathrm{A}_{2 \mathrm{u}}(46)$ phonon mode, which is in the $\mathrm{THz}$ range and 
thus significantly above the detection limit of the resonance measurements.

While the compatibility of the symmetry of ferroic ordering of $\mathrm{Cu} M_{z^{2}}$ magnetic quadrupoles with neutron measurements has already been proposed [18,30], here we show that such magnetoelectric multipoles also resolve the paradox between the values of local magnetic dipole moments inferred from interpreting the neutron data in terms of magnetic dipole scattering, and the much smaller values measured from the local probe NMR and $\mu$ SR methods. Detailed analysis of the neutron scattering data for YBCO showed that the signal could be interpreted in terms of magnetic dipole moments on the oxygen atoms with a maximum magnitude of $\sim 0.1 \mu_{B}$ [3], apparently incompatible with the much lower limits set by NMR and $\mu \mathrm{SR}$. When we perform a DFT calculation with the oxygen dipole moments constrained to this value, we generate an atomic-site magnetic quadrupole on $\mathrm{Cu}$ that has a magnitude close to that generated by our quasistatic mechanism. (Note, however, that, as we stated previously, it is energetically costly to force magnetic dipole moments onto the closed-shell oxygen atoms.) We propose, therefore, that the neutron measurements are sensitive directly to the magnetoelectric multipoles, and the apparent inconsistencies between the various experimental methods are an artifact of an attempt to map the magnetoelectric multipolar ordering onto a dipolar picture.

A defining characteristic of the pseudogap phase is, of course, the existence of a pseudogap. We searched for this by monitoring the change in the density of states on freezing in the $\mathrm{A}_{2 \mathrm{u}}$ (46) pattern of atomic displacements with progressively increasing amplitude, and indeed found a reduction in the density of states at the Fermi level $N\left(E_{F}\right)$, which we show in Fig. 7(a). Note that our suggested mode at $46 \mathrm{meV}$ causes significant reductions of the electron count at the Fermi energy. This reduction will in turn reduce the electronic specific heat and conductance, as observed in experiment [53]. Since our order parameter is not a conventional static Landau-type order parameter, however, there is no associated soft mode and we do not expect a discontinuity in specific heat. Again, this is compatible with experimental measurements.

Perhaps the most compelling evidence for the mechanism proposed here is the recent observation using neutron scattering of an Ising-type excitation at $\sim 40 \mathrm{meV}$ in $\mathrm{Hg}-1201$ at the onset to the pseudogap phase [29]. We assign this Ising-type excitation to our coupled spin $\mathrm{A}_{2 \mathrm{u}}(46)$ phonon mode responsible for the quadrupolar order; our calculations of the doping dependence of the phonon frequencies indicate that the difference in energy due to the higher doping level $\delta_{\text {expt }} \approx 0.11$ used in the experiment. Intriguingly, as shown in Fig. 7(b), we also find a strong doping dependence of the spin-phonon coupling, with the spin-phonon coupling of the $A_{2 u}(46)$ mode approaching zero as doping is increased beyond the optimally doped range, in the same manner as $T^{*}$.

\section{SUMMARY AND DISCUSSION}

In summary, we have shown that a quasistatic ordering of magnetoelectric multipoles mediated by coupling between paramagnetic spin moments on the $\mathrm{Cu}$ ions and a polar lattice phonon provides a candidate order parameter for the
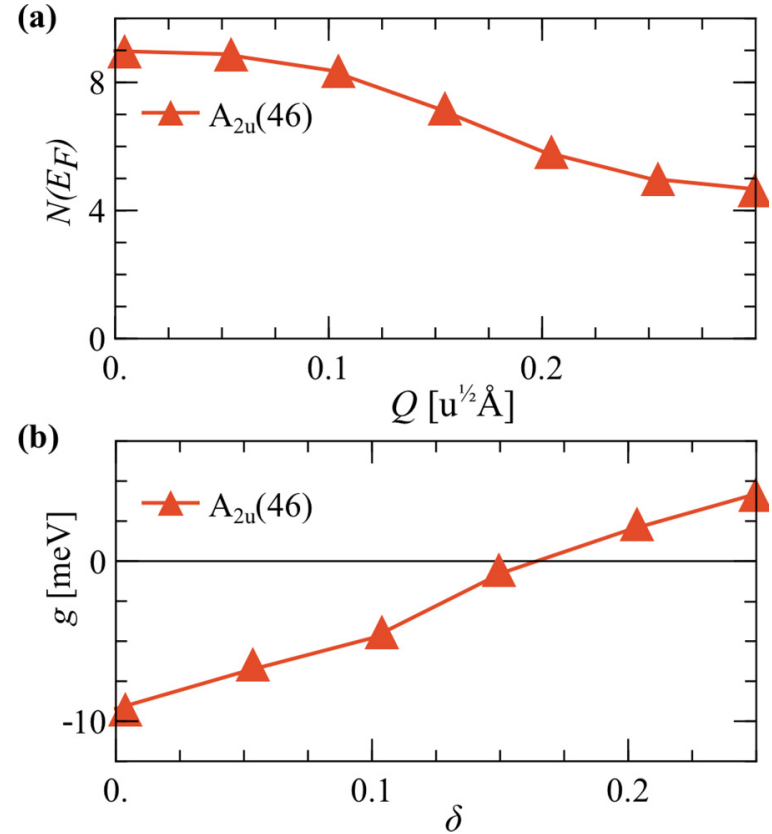

FIG. 7. (a) Density of states at the Fermi energy $\left[N\left(E_{F}\right)\right]$ as a function of the amplitude of the polar $\mathrm{A}_{2 \mathrm{u}}(46)$ mode. Note that the reduction of $N\left(E_{F}\right)$ is symmetric against inversion of the amplitude. (b) Spin-phonon coupling constant of the $\mathrm{A}_{2 \mathrm{u}}(46)$ phonon mode as a function of doping $\delta$.

pseudogap phase of the high- $T_{c}$ cuprates. This candidate mechanism is, to our knowledge, consistent with all known experimental data. In addition, it captures the spirit of the earlier orbital-current models as well as the analysis of neutron scattering in terms of magnetoelectric multipoles, while providing a microscopic origin for the behavior. There remain many open questions regarding this quasistatic magnetoelectric quadrupolar ordering that require further experimental measurement and theoretical analysis.

The first is whether such a dynamically varying ordering that fluctuates with a net nonzero value constitutes an order parameter and, in turn, whether its onset should be described as a phase transition. It is certainly the case that the appearance of the quadrupolar order corresponds to a lowering of the symmetry as both time-reversal and space-inversion symmetries are broken. The microscopic coupling mechanism, however, does not fit straightforwardly into the usual Landau description of spontaneous symmetry-breaking phase transitions since the order parameter is generated by coupled fluctuations. To the extent that it is associated with a well-defined symmetry lowering, it has characteristics of a phase transition. In addition, it has features of a ferroic transition, with multiple equivalent ground states that can in principle form domains. The absence of a soft mode or divergence of the specific heat, however, are clearly unconventional.

A second question is how the dynamical order competes and/or coexists with other orders. For example, most holedoped cuprates show a charge-density wave (CDW) order [54], which emerges within the pseudogap region of the phase diagram in a region substantially below $T^{*}$ but above the superconducting dome. While it is now widely accepted, based in part on their different onset temperatures, that the CDW 
phase is distinct from the pseudogap phase [54], an interaction or competition between their orders is likely. Indeed, a recent $\mathrm{x}$-ray scattering study of $\mathrm{YBa}_{3} \mathrm{CuO}_{6+\delta}$ in strong applied magnetic fields [55] showed a pronounced enhancement of the CDW phase into the pseudogap region of the phase diagram in the presence of the magnetic field. Since strong magnetic fields also suppress spin fluctuations, we anticipate an accompanying suppression of magnetoelectric multipolar ordering through the dynamical spin-phonon coupling mechanism. An exploration of such an effect will form the topic of future work.

Another obvious question is how to measure the proposed effect. One possibility could be a direct mapping of the associated internal quadrupolar magnetic field using $\mu \mathrm{SR}$, or the associated field gradients using NMR or NQR; in particular, our initial calculations in this direction suggest that these have a strong doping dependence. Such a measurement could also distinguish quasistatic multipoles from those generated by a purely orbital-current mechanism, which, as we mentioned earlier, carry also a magnetic dipole contribution. Perhaps a more convincing distinction between the orbital-current mechanism and quasistatic quadrupoles could be revealed from studies of oxygen isotope effects, to which our model should be rather sensitive and the orbital currents not. Measurement of the E1-E2 pre-edge transition with magnetochiral diffraction would also provide evidence for the quadrupolar order although not for its origin. A successful experiment, however, would require a single-domain quadrupolar state. We expect that the length scale of the domains should be set by the correlation lengths of the phonon and spin fluctuations, although simultaneous electric and magnetic fields should in principle provide a conjugate field for achieving a singledomain state.

We make no statement at this stage as to whether the quasistatic quadrupolar ordering competes with the superconducting state or is a precursor to it. If the latter, it could harmoniously resolve the ongoing dispute as to whether the pairing mechanism is spin or lattice driven since its existence requires a coupling of the two. A possible experiment to address this question would be optical pumping of the associated $\mathrm{A}_{2 \mathrm{u}}(46)$ phonon mode, in a similar manner as used recently in Refs. [56,57] to study the origin of superconductivity in $\mathrm{YBCO}$. The $46-\mathrm{meV}$ frequency is in a particularly inconvenient range in terms of experimental accessibility, however. In the meantime, this intriguing hint of a time and space odd order parameter suggests that perhaps the family of multiferroic and magnetoelectric compounds should receive more attention as potential superconductors.

Finally, we note that the model Hamiltonian of Eq. (4) is not restricted to polar or zone-center modes, and coupling to other modes, or to non-zone-center phonons could give quasistatic ordering of other elusive order parameters, such as charge ordering or stripes, that are associated with exotic superconductivity.

\section{ACKNOWLEDGMENTS}

This work was supported financially by the ETH Zürich (M.J.A.F. and N.A.S.), by the ERC Advanced Grant program, No. 291151 (M.F. and N.A.S.), by the Max Rössler Prize of the ETH Zürich (N.A.S.), and by the Sinergia program of the Swiss National Science Foundation Grant No. CRSII2_147606/1 (F.T., N.A.S., and U.S.). This work was supported by a grant from the Swiss National Supercomputing Centre (CSCS) under Project No. ID s624. We thank L. Nordström for assistance with developments in the ELK code, and B. Batlogg, P. Bourges, S. DiMatteo, P. Gambardella, A. Georges, J. Haase, A. Kapitulnik, D. Khalyavin, S. Kivelson, S. Lovesey, and J. Zaanen for fruitful discussions and valuable feedback on the paper.
[1] C. M. Varma, Nature (London) 468, 184 (2010).

[2] W. W. Warren, Jr., R. E. Walstedt, G. F. Brennert, R. J. Cava, R. Tycko, R. F. Bell, and G. Dabbagh, Phys. Rev. Lett. 62, 1193 (1989).

[3] B. Fauqué, Y. Sidis, V. Hinkov, S. Pailhès, C. T. Lin, X. Chaud, and P. Bourges, Phys. Rev. Lett. 96, 197001 (2006).

[4] A. Shekhter, B. J. Ramshaw, R. Liang, W. N. Hardy, D. A. Bonn, F. F. Balakirev, R. D. McDonald, J. B. Betts, S. C. Riggs, and A. Migliori, Nature (London) 498, 75 (2013).

[5] S. Strassle, B. Graneli, M. Mali, J. Roos, and H. Keller, Phys. Rev. Lett. 106, 097003 (2011).

[6] Y. Tokunaga, K. Ishida, K. Magishi, S. Ohsugi, G. q. Zheng, Y. Kitaoka, K. Asayama, A. Iyo, K. Tokiwa, and H. Ihara, Phys. B (Amsterdam) 259, 571 (1999).

[7] J. E. Sonier, J. H. Brewer, R. F. Kiefl, R. I. Miller, and G. D. Morris, Science 292, 1692 (2001).

[8] A. Kaminski, S. Rosenkranz, H. M. Fretwell, J. C. Campuzano, Z. Li, H. Raffy, W. G. Cullen, H. You, C. G. Olson, C. M. Varma et al., Nature (London) 416, 610 (2002).
[9] S. V. Borisenko, A. A. Kordyuk, A. Koitzsch, M. Knupfer, J. Fink, H. Berger, and C. T. Lin, Nature (London) 431, 1 (2004).

[10] V. Arpiainen, A. Bansil, and M. Lindroos, Phys. Rev. Lett. 103, 067005 (2009).

[11] M. R. Norman, A. Kaminski, S. Rosenkranz, and J. C. Campuzano, Phys. Rev. Lett. 105, 189701 (2010).

[12] J. Xia, E. Schemm, G. Deutscher, S. A. Kivelson, D. A. Bonn, W. N. Hardy, R. Liang, W. Siemons, G. Koster, M. M. Fejer et al., Phys. Rev. Lett. 100, 127002 (2008).

[13] G. J. MacDougall, A. A. Aczel, J. P. Carlo, T. Ito, J. Rodriguez, P. L. Russo, Y. J. Uemura, S. Wakimoto, and G. M. Luke, Phys. Rev. Lett. 101, 017001 (2008).

[14] J. E. Sonier, V. Pacradouni, S. A. Sabok-Sayr, W. N. Hardy, D. A. Bonn, R. Liang, and H. A. Mook, Phys. Rev. Lett. 103, 167002 (2009).

[15] S. Strässle, J. Roos, M. Mali, H. Keller, and T. Ohno, Phys. Rev. Lett. 101, 237001 (2008).

[16] C. M. Varma, Phys. Rev. B 55, 14554 (1997). 
[17] S. W. Lovesey, J. Phys.: Condens. Matter 26, 356001 (2014).

[18] S. W. Lovesey, D. D. Khalyavin, and U. Staub, J. Phys.: Condens. Matter 27, 292201 (2015).

[19] N. A. Spaldin, M. Fechner, E. Bousquet, A. Balatsky, and L. Nordström, Phys. Rev. B 88, 094429 (2013).

[20] L. Mangin-Thro, Y. Sidis, A. Wildes, and P. Bourges, Nat. Commun. 6, 7705 (2015).

[21] J. Orenstein, Phys. Rev. Lett. 107, 067002 (2011).

[22] N. Barisic, Y. Li, X. Zhao, Y.-C. Cho, G. Chabot-Couture, G. Yu, and M. Greven, Phys. Rev. B 78, 054518 (2008).

[23] A. Yamamoto, W. Z. Hu, and S. Tajima, Phys. Rev. B 63, 024504 (2000).

[24] Q. Huang, J. W. Lynn, Q. Xiong, and C. W. Chu, Phys. Rev. B 52, 462 (1995).

[25] Y. Itoh, T. Machi, A. Fukuoka, K. Tanabe, and H. Yasuoka, J. Phys. Soc. Jpn. 65, 3751 (1996).

[26] A. M. Mounce, S. Oh, J. A. Lee, W. P. Halperin, A. P. Reyes, P. L. Kuhns, M. K. Chan, C. Dorow, L. Ji, D. Xia et al., Phys. Rev. Lett. 111, 187003 (2013).

[27] H. Uchiyama, W. Z. Hu, A. Yamamoto, S. Tajima, K. Saiki, and A. Koma, Phys. Rev. B 62, 615 (2000).

[28] Y. Li, V. Balédent, N. Barišić, Y. Cho, B. Fauqué, Y. Sidis, G. Yu, X. Zhao, P. Bourges, and M. Greven, Nature (London) 455, 372 (2008).

[29] Y. Li, G. Yu, M. K. Chan, V. Balédent, Y. Li, N. Barišić, X. Zhao, K. Hradil, R. A. Mole, Y. Sidis et al., Nat. Phys. 8, 404 (2012).

[30] S. W. Lovesey and D. D. Khalyavin, Journal of Physics: Condensed Matter 27, 49 (2015).

[31] P. Bourges (private communication).

[32] J. Yang, J. Hwang, E. Schachinger, J. P. Carbotte, R. P. S. M. Lobo, D. Colson, A. Forget, and T. Timusk, Phys. Rev. Lett. 102, 027003 (2009).

[33] W. Tabis, Y. Li, M. Le Tacon, L. Braicovich, A. Kreyssig, M. Minola, G. Dellea, E. Weschke, M. J. Veit, M. Ramazanoglu et al., Nat. Commun. 5, 1 (2014).

[34] S. Di Matteo and M. R. Norman, Phys. Rev. B 85, 235143 (2012).

[35] V. M. Yakovenko, Phys. B (Amsterdam) 460, 159 (2015).

[36] V. I. Anisimov, J. Zaanen, and O. K. Andersen, Phys. Rev. B 44, 943 (1991).

[37] V. I. Anisimov, F. Aryasetiawan, and A. I. Liechtenstein, J. Phys.: Condens. Matter 9, 767 (1997).

[38] D. Wu, Q. Zhang, and M. Tao, Phys. Rev. B 73, 235206 (2006).
[39] B. Himmetoglu, R. M. Wentzcovitch, and M. Cococcioni, Phys. Rev. B 84, 115108 (2011).

[40] G. Kresse and J. Furthmüller, Phys. Rev. B 54, 11169 (1996).

[41] P. E. Blochl, Phys. Rev. B 50, 17953 (1994).

[42] LAPW ELK code, http://elk.sourceforge.net

[43] C. Ambrosch-Draxl, P. Süle, H. Auer, and E. Y. Sherman, Phys. Rev. B 67, 100505 (2003).

[44] T. Thonhauser, H. Auer, E. Y. Sherman, and C. Ambrosch-Draxl, Phys. Rev. B 69, 104508 (2004).

[45] C. Ambrosch-Draxl, E. Y. Sherman, H. Auer, and T. Thonhauser, Phys. Status Solidi B 241, 1199 (2004).

[46] C. Ambrosch-Draxl, E. Y. Sherman, H. Auer, and T. Thonhauser, Phys. Rev. Lett. 92, 187004 (2004).

[47] C. Ambrosch-Draxl and E. Y. Sherman, Phys. Rev. B 74, 024503 (2006).

[48] D. Muñoz, F. Illas, and I. de P. R. Moreira, Phys. Rev. Lett. 84, 1579 (2000).

[49] M. d'Astuto, A. Mirone, P. Giura, D. Colson, A. Forget, and M. Krisch, J. Phys.: Condens. Matter 15, 8827 (2003).

[50] H. Uchiyama, A. Q. R. Baron, S. Tsutsui, Y. Tanaka, W.-Z. Hu, A. Yamamoto, S. Tajima, and Y. Endoh, Phys. Rev. Lett. 92, 197005 (2004).

[51] We use the numerical solver for differential equations implemented in the Mathematica [58] software package. For the friction constant $\gamma$ we use the lifetime of the phonon $(\tau=1 / \gamma)$, which we set to a value of $0.25 \mathrm{ps}$. The numerical solution requires two initial conditions, which we take to be a nondisplaced mode $[Q(0)=0]$ with a finite velocity $\left[Q^{\prime}(0) \neq 0\right]$.

[52] B. B. Krichevtsov, V. V. Pavlov, R. V. Pisarev, and V. N. Gridnev, J. Phys.: Condens. Matter 5, 8233 (1993).

[53] T. Timusk and B. Statt, Rep. Prog. Phys. 62, 61 (1999).

[54] B. Keimer, S. A. Kivelson, M. R. Norman, S. Uchida, and J. Zaanen, Nature (London) 518, 179 (2015).

[55] S. Gerber, H. Jang, H. Nojiri, S. Matsuzawa, H. Yasumura, D. A. Bonn, R. Liang, W. N. Hardy, Z. Islam, A. Mehta et al., Science 350, 949 (2015).

[56] W. Hu, S. Kaiser, D. Nicoletti, C. R. Hunt, I. Gierz, M. C. Hoffmann, M. Le Tacon, T. Loew, B. Keimer, and A. Cavalleri, Nat. Mater. 13, 705 (2014).

[57] R. Mankowsky, A. Subedi, M. Först, S. O. Mariager, M. Chollet, H. T. Lemke, J. S. Robinson, J. M. Glownia, M. P. Minitti, A. Frano et al., Nature (London) 516, 71 (2014).

[58] Mathematica version 10.2.0.0, http://www.wolfram.com 